\title{
X-ray Flare Spectra from the DIOGENESS Spectrometer and Its Concept Applied to ChemiX on the Interhelioprobe Spacecraft
}

\author{
Janusz Sylwester ${ }^{1}$ - Zbigniew Kordylewski ${ }^{1}$ - Stefan Płocieniak ${ }^{1} \cdot$ Marek Siarkowski $^{1}$. \\ Mirosław Kowaliński ${ }^{1}$ - Stanisław Nowak ${ }^{1}$ - Witold Trzebiński ${ }^{1}$ - Marek Śtęślicki ${ }^{1}$. \\ Barbara Sylwester $^{1} \cdot$ Eugeniusz Stańczyk $^{1} \cdot$ Ryszard Zawerbny $^{1} \cdot \dot{Z Z a n e t a ~ S z a f o r z ~}^{1}$. \\ Kenneth J.H. Phillips ${ }^{2}$ - František Fárník ${ }^{3}$ - Anatolyi Stepanov ${ }^{4}$
}

Received: 10 September 2014 / Accepted: 16 December 2014 / Published online: 20 January 2015

(C) The Author(s) 2015. This article is published with open access at Springerlink.com

\begin{abstract}
The DIOGENESS X-ray crystal spectrometer on the CORONAS-F spacecraft operated only for a single month (25 August to 17 September) in 2001, but in its short lifetime obtained one hundred and forty high-resolution spectra of eight solar flares with GOES importance ranging from C9 to X5. The instrument included four scanning flat crystals with wavelength ranges covering the regions of Si XIII (6.65 $), \mathrm{S}$ XV (5.04 $\AA$ ), and Ca XIX (3.18 A) X-ray lines and associated dielectronic satellites. Two crystals covering the Ca XIX lines were oriented in a "dopplerometer" manner, i.e. such that spatial and spectral displacements, both of which commonly occur in flares, can be separated. We describe the DIOGENESS spectrometer and the spectra obtained during flares that include lines not hitherto seen from spacecraft instruments. An instrument with a very similar concept is currently being built for the two Russian Interhelioprobe spacecraft that are scheduled for launch in 2020 and 2022 and will make a near-encounter (perihelion $\sim 0.3 \mathrm{AU}$ ) with the Sun in its orbit. We outline the results that are likely to be obtained.
\end{abstract}

Solar and Stellar Flares: Observations, Simulations, and Synergies

Guest Editors: Lyndsay Fletcher and Petr Heinzel

E. Stańczyk and R. Zawerbny are deceased.

J. Sylwester

js@cbk.pan.wroc.pl

K.J.H. Phillips

kennethjhphillips@yahoo.com

F. Fárník

ffarnik@asu.cas.cz

\section{A. Stepanov}

as@izmiran.ru

1 Space Research Centre, Polish Academy of Sciences, Kopernika 11, 51-622 Wrocław, Poland

2 Earth Sciences Department, Natural History Museum, London SW7 5BD, UK

3 Astronomical Institute, The Czech Academy of Sciences, 25165 Ondřejov, Czech Republic

4 Institute of Terrestrial Magnetism and Radiowave Propagation (IZMIRAN), Troisk, Moscow, Russia 
Keywords Flares, Spectrum · Spectral Line, Intensity and Diagnostics $\cdot$ Spectrum, X-ray

\section{Introduction}

Scanning X-ray flat crystal spectrometers on rockets and spacecraft have been used to observe flares and non-flaring active regions for many years, and the resulting spectra have helped our understanding of the energetics and physical characteristics of the emitting regions and the mechanism of flare energy release. Instruments with an accurate absolute intensity calibration have also enabled element abundances in the corona to be found. The rapidly developing initial phases of flares, in which high-temperature plasma is ejected from localised sources such as the footpoints of magnetic flux tubes, requires X-ray spectrometers that can acquire and store data on very short time-scales. This has led to the application of bent crystal spectrometers with position-sensitive detectors for solar-dedicated spacecraft. Early examples include those on the Solar Maximum Mission (BCS or Bent Crystal Spectrometer: Acton et al., 1980), Yohkoh (BCS or Bragg Crystal Spectrometer: Culhane et al., 1991), and CORONAS-F (RESIK or REntgenovsky Spektrometr s Izognutymi Kristalami: Sylwester et al., 2005).

Bent crystal spectrometers have the advantage of obtaining spectra over their wavelength ranges instantaneously in a particular data-gathering interval, typically a few seconds, and also of generally having a higher sensitivity than flat crystal spectrometers. However, to observe the initial phases of flares, spectra from bent crystal spectrometers, especially those without a fine collimator that reduces their sensitivity, are subject to a confusion of spectral and spatial blurring without being able to untangle the two unless independent measurements are available. This difficulty may be overcome with the simultaneous observation of flares with a pair of crystals oriented in such a way that the spatial displacements occur to longer wavelengths for one of them and towards shorter wavelengths for the other. This "dopplerometer" mode was proposed for an instrument initially launched on a Vertical rocket in the Russian space programme by the Space Research Centre of the Polish Academy of Sciences in 1981. A later version, called DIOGENESS (DIagnostics Of Global ENErgy Sources and Sinks) was built by SRC to be included in the Russian CORONAS-I spacecraft in the mid-1990s and, following the failure of the instrument, for the subsequent CORONAS-F spacecraft, which was operational from 2001 to 2006. Four scanning flat crystals were included to observe the vicinity of intense lines of high-temperature ions - Si XIII $(6.65 \AA), \mathrm{S} \times \mathrm{V}(5.04 \AA)$, and Ca XIX $(3.18 \AA)$ - which are prominent in flare and some active region spectra, with two of the crystals observing the Ca XIX lines in a dopplerometer arrangement.

The CORONAS-F spacecraft was launched on 31 July 2001 and worked successfully until the end of 2006, with RESIK and DIOGENESS included in the instrument package. While RESIK obtained spectra until May 2003, DIOGENESS operated for only a few weeks because a fault in the scanning drive mechanism occurred on 17 September 2001. However, eight flares with GOES importance up to X5.5 were observed and one hundred and forty spectra were obtained in four wavelength channels. Detailed analysis was delayed for some years while the more extensive RESIK data set were examined. In the meantime, another version of DIOGENESS, called ChemiX, is being built to be included in the instrument package on two Russian Interhelioprobe spacecraft, scheduled for launch in 2020 and 2022, which will eventually orbit the Sun in highly elliptical paths, with $30^{\circ}$ inclination to the ecliptic plane, bringing them to within 0.3 astronomical units from the Sun. It is appropriate, therefore, to describe the DIOGENESS and ChemiX instruments in some detail at this time, 
even though the two earlier versions were launched some years ago. In particular, it is of interest to describe the DIOGENESS spectra, which have particularly good resolution and allow the flare dynamics to be determined as well as investigation of lines not previously noted in solar spectra.

In this article, we describe the DIOGENESS instrument and some details of the CORONAS-F spacecraft and operations (Section 2), the flare spectra obtained in its operating lifetime, including a list of the lines observed (Section 3), and describe the forthcoming ChemiX instrument that is scheduled to fly on the Interhelioprobe missions (Section 4).

\section{CORONAS-F and the DIOGENESS Instrument}

\subsection{Spacecraft and Performance}

CORONAS- $F$ was the second of three Russian spacecraft designed to observe various solar phenomena in the period 1994 - 2009, with thirteen instruments studying global oscillations, variations in ultraviolet, X-ray, and $\gamma$-ray, emission, and particle emission. The spacecraft was launched into a near-circular orbit around Earth with an altitude range of $501-549 \mathrm{~km}$. The orbital plane was inclined at $82^{\circ} .5$ to the equator, and its initial period was 94.9 minutes. Intervals of uninterrupted solar observation were possible for up to 20 days on two occasions during a year, while at other times the spacecraft night-time periods lasted no more than 35 minutes. Solar X-ray observations were also interrupted by passages through the auroral oval particle zones near the Earth's magnetic poles and the South Atlantic Anomaly. Spacecraft pointing at the Sun was achieved with a 15 -arcminute precision.

The instrument package is described by Oraevsky and Sobelman (2002). The RESIK and DIOGENESS instruments were built by teams led by the Space Research Centre, Polish Academy of Sciences, in Wrocław, Poland. RESIK has been described by Sylwester et al. (2005). It was a bent crystal spectrometer with four channels observing X-ray flare and non-flaring active regions in the range 3.3-6.1 $\AA$, which included emission lines of highly ionized Si, S, Cl, Ar, and $\mathrm{K}$ ions. An absolute flux calibration of $\sim 20 \%$ precision was achieved for the instrument, allowing element abundances to be determined. These estimates have been discussed in several publications: see Sylwester et al. (2014) and references therein. RESIK operated from the time of spacecraft launch until May 2003, when the instrument power supply failed.

\subsection{DIOGENESS: Instrument and Performance}

The DIOGENESS instrument, in contrast to RESIK, had a lifetime of only a few weeks, but the instrumental concept, including that of the dopplerometer, was adequately verified. The construction of the instrument was outlined by Płocieniak et al. (2002) and Siarkowski et al. (2002). DIOGENESS had four channels covering wavelength ranges in the vicinity of intense lines of highly ionized $\mathrm{Si}, \mathrm{S}$, and $\mathrm{Ca}$ lines visible in solar flares. Two quartz crystals covered the Ca XIX lines (channels 1 and 4), and ADP and beryl crystals (channels 2 and 3) covered the S XV and Si XIII lines, respectively. Table 1 lists details of the wavelength ranges and crystals. The mono-crystals were flat and mounted on a common rotatable shaft, so a scanning motion back and forth of the shaft allowed X-rays with various wavelengths $\lambda$ to be diffracted according to Bragg's diffraction law,

$$
n \lambda=2 d \sin \theta,
$$


Table 1 DIOGENESS instrument channels.

\begin{tabular}{lllll}
\hline Channel & 1 & 2 & 3 & 4 \\
\hline Crystal & Quartz & ADP & Beryl & Quartz \\
Diffracting plane & $10 \overline{1} 1$ & 101 & 1010 & $10 \overline{1} 1$ \\
$2 d$ spacing $(\AA)^{\mathrm{a}}$ & 6.6855 & 10.5657 & 15.9585 & 6.6875 \\
Central wavelength $(\AA)$ & 3.1781 & 5.0374 & 6.6488 & 3.1781 \\
Principal lines in range & Ca XIX & S XV & Si XIII & Ca XIX \\
Min. of wavelength range $(\AA)$ & 3.1436 & 4.9807 & 6.1126 & 2.9601 \\
Max. of wavelength range $(\AA)$ & 3.3915 & 5.3721 & 6.7335 & 3.2123 \\
Crystal reflectivity $(\mu \mathrm{rad})^{\mathrm{a}}$ & 91 & 91 & 15 & 90 \\
Rocking curve $\left(\mathrm{FWHM}\right.$, arcsec) $^{\mathrm{a}}$ & 24.1 & 68.1 & 94.1 & 25.6 \\
\hline
\end{tabular}

${ }^{\text {a}}$ Prelaunch measurement.

where $d$ is the crystal lattice spacing, $\theta$ the angle of incidence, and $n$ the diffraction order. Only first-order $(n=1)$ spectra were recorded. The angular range of the crystal shaft was 140 arcmin, giving the wavelength ranges specified in Table 1. The spectral resolution of each crystal is defined by the rocking curve, whose values (FWHM) are given in arcsec in Table 1 . The instrument was uncollimated, but as flares generally have small angular extents (typically a few arcmin), the emission region can be considered to be a point source, so a collimator is generally not necessary. The diffracted X-rays are registered by double proportional counters with beryllium entrance windows of $145 \mu \mathrm{m}$ thickness and are filled with argon gas with a small admixture of carbon dioxide quenching gas at $\sim 0.5$ atmospheric pressure. An $\mathrm{Fe}^{55}$ source (energy $5.9 \mathrm{keV}$ ) illuminates the other, non-solar proportional counter section, so that the detector's electronic system energy gain could be checked. A multi-slit collimator with soft X-ray detectors scanning in the direction of the spectrometer's dispersion was also included to give spatial context information, but unfortunately, this part of the instrument failed early in the mission.

For channels 2 and 3, the S XV and Si XIII lines were repeatedly scanned over the period of a flare in the direction of alternately increasing and decreasing wavelengths. Figure 1 shows the arrangement for channels 1 and 4, which viewed the Ca XIX lines with identical quartz crystals cut from the same parent mono-crystal at an angle $\alpha$ between the diffracting planes of the crystals, the so-called dopplerometer mode. Solar X-rays are incident on each crystal in the manner illustrated in the figure (left panel). As the two crystals on their common shaft are rotated, solar X-ray lines are successively recorded by each channel from the point-like flare on the Sun, as shown in the right panel of Figure 1. The angle $\alpha$ was selected such that the Ca XIX resonance line $w$ emitted by flare emission with zero radial velocity was observed simultaneously, its theoretical value being $56^{\circ} .7416$. (In practice, owing to co-alignment uncertainty, the angle $\alpha$ was set to $56^{\circ} .8000$.) In Figure 1 , the two occasions when the vicinity of the Ca XIX $w$ line was scanned simultaneously in the sense of increasing wavelengths (red scan in the figure) and decreasing wavelengths (blue scan) are shown. The horizontal axis indicates the stepper drive's address rather than wavelength. For zero radial velocity of the flare source, the Ca XIX $w$ line would be coincident for each scan, but because of the presence of an approach velocity (such wavelength shifts have been commonly attributed to "chromospheric evaporation" or upward convection of the emitting plasma), the lines are separated in each scan. The linearity of the scanning mechanism was checked during the spacecraft mission using strain-gauge systems. 

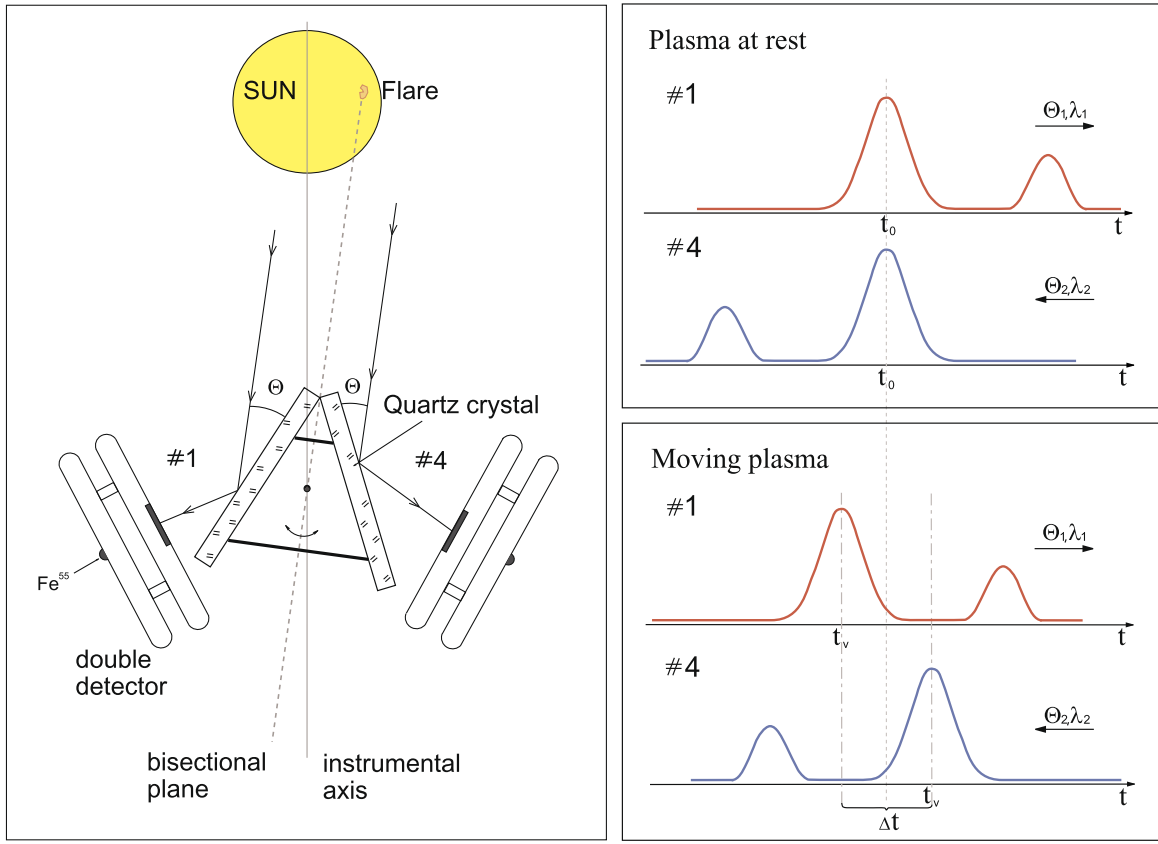

Figure 1 Left panel: Principle of the DIOGENESS dopplerometer. Solar X-rays are incident on two identical quartz crystals oriented as shown. The diffracted rays around the wavelengths of the Ca XIX lines near $3.177 \AA$ are detected by double proportional counters (labelled 1 and 4 in the figure). The detector energy gain is checked with $\mathrm{Fe}^{55}$ sources at the rear of each detector. Right panel: Resulting Ca XIX spectra, showing the more intense resonance $(w)$ and less intense intercombination $(x, y)$ lines (see Table 3). In the upper panel the flare plasma is assumed to be at rest with respect to the instrument, and the Ca XIX $w$ (resonance) lines are coincident (for detector 1 wavelength $\lambda$ increases to the right, for detector 4 it increases to the left). In the lower panel the flare plasma is moving with approaching velocity (as is usually the case with flare impulsive phases), so the line wavelengths are displaced to shorter wavelengths in opposite directions. Any shifts due to spatial movement across the line of sight will be seen as wavelength shifts in identical directions.

The effective areas of the four DIOGENESS channels (see Figure 2) were measured before spacecraft launch and are the product of detector efficiency, filter transmission, crystal integrated reflectivity, and projected area of the detector window as seen from the Sun.

\section{DIOGENESS Observations}

\subsection{Flare Spectra}

Eight intense flares were observed during the lifetime of DIOGENESS; see Table 2 for details of these flares including times, standard IAU flare notation, GOES and H $\alpha$ importance, and heliographic location. One hundred and forty spectra were obtained in the four channels for these events.

The X5 flare on 25 August 2001 was observed to great advantage by DIOGENESS, from the earliest pre-flare state including a small precursor flare (16:17 UT) and the impulsive phase at around 16:30 - 16:32 UT, also seen with the Yohkoh Hard X-ray Telescope (HXT), until late in the decay phase, a total of nearly two hours. Figure 3 shows the light curves 

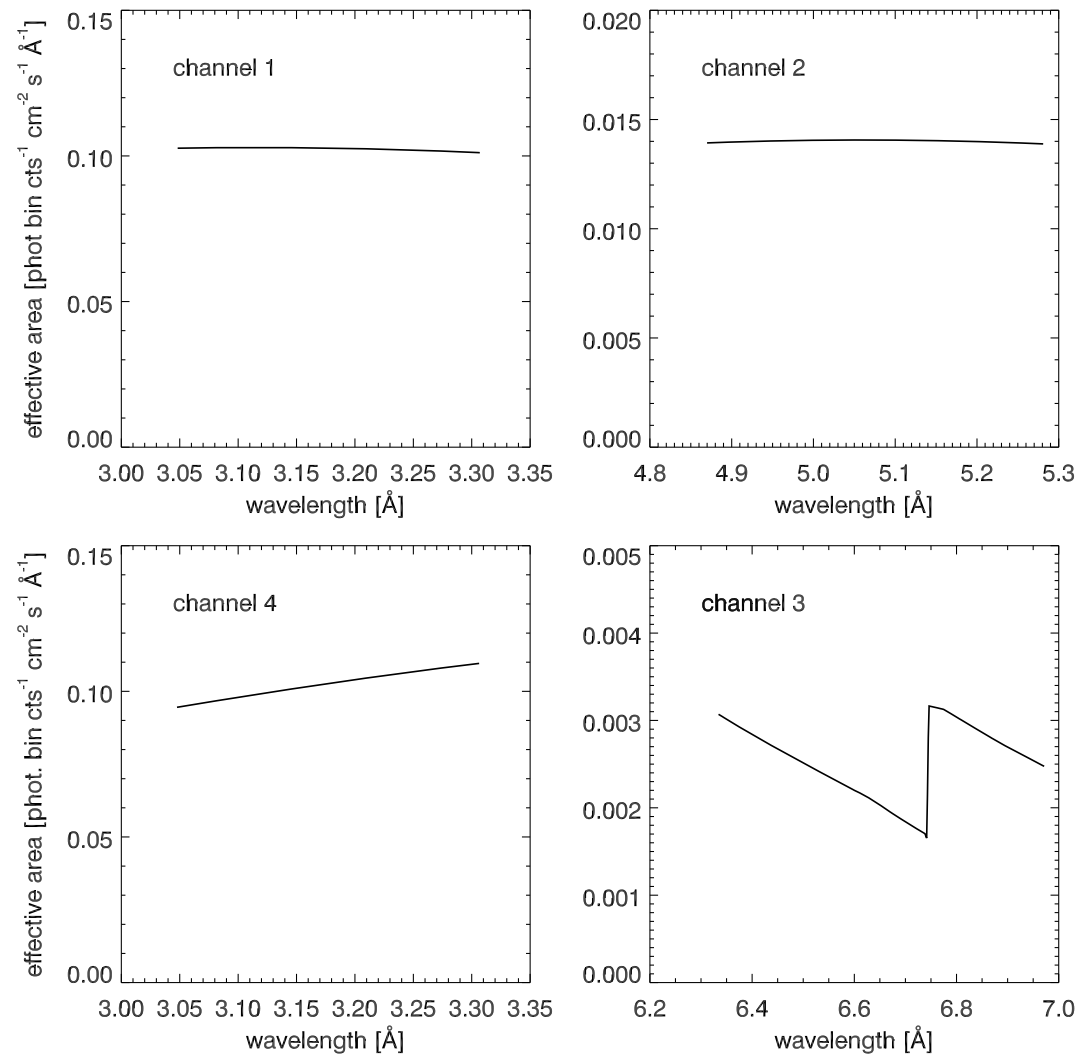

Figure 2 Effective areas of the four channels of DIOGENESS. The units are photon bin count $^{-1}$ $\mathrm{cm}^{-2} \mathrm{sec}^{-1} \AA^{-1}$. The channel numbers are indicated in the legend.

Table 2 Flares observed by DIOGENESS.

\begin{tabular}{llllll}
\hline Date (2001) & UT of peak & Flare notation & $\begin{array}{l}\text { GOES } \\
\text { importance }\end{array}$ & $\begin{array}{l}\text { H } \alpha \\
\text { importance }\end{array}$ & Location $^{\text {a }}$ \\
\hline Aug. 25 & $16: 45$ & SOL2001-08-25T16:45 & X5.3 & 3B & S17E34 \\
Aug. 30 & $17: 57$ & SOL2001-08-30T17:57 & M1.5 & 2N & S21W28 \\
Sept. 2 & $06: 02$ & SOL2001-09-02T06:02 & M1.3 & $1 \mathrm{~F}$ & S17W66 \\
Sept. 2 & $13: 48$ & SOL2001-09-02T13:48 & M3.0 & 2N & S21W65 \\
Sept. 3 & $01: 58$ & SOL2001-09-03T01:58 & C9.0 & - & S17E90 \\
Sept. 3 & $17: 16$ & SOL2001-09-03T17:16 & M1.1 & - & N10W06 \\
Sept. 3 & $18: 41$ & SOL2001-09-03T18:41 & M2.5 & - & S26E90 \\
Sept. 16 & 03:53 & SOL2001-09-16T03:53 & M5.6 & 2N & S29W54 \\
\hline
\end{tabular}

${ }^{a}$ No $\mathrm{H} \alpha$ records for the Sept. 3 flares; locations from Yohkoh HXT images.

from the two GOES channels and the total emission in DIOGENESS channel 1 (Ca XIX channel). The DIOGENESS light curve shows the Ca XIX line emission because the Bragg diffraction condition (Equation (1)) was successively satisfied for the Ca XIX emission lines 


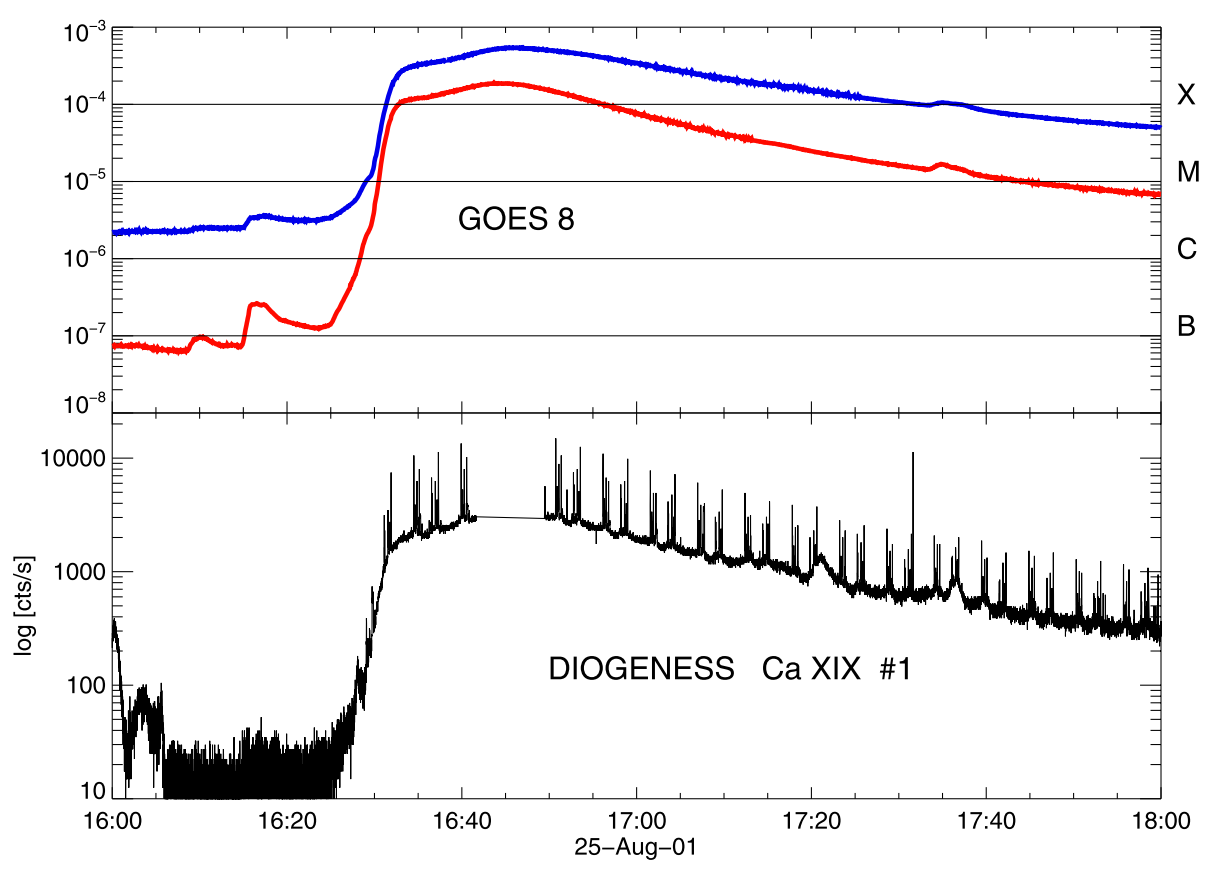

Figure 3 Top: Light curves of the two channels of GOES (red - 0.5- $\AA$; blue $-1-8 \AA$ ), irradiance scale in $\mathrm{W} \mathrm{m}^{-2}$ on the left axis (GOES importance scale on the right axis) for the 25 August 2001 flare. Lower panel: DIOGENESS Ca XIX count rate (logarithmic scale), showing the scans through the Ca XIX lines as the crystals rocked back and forth (time of scan $140 \mathrm{~s}$ ). A passage of the CORONAS-F spacecraft through the auroral oval radiation belts before the flare occurred around 16:00-16:10 UT.

a total of $\sim 30$ scans, i.e. approximately $140 \mathrm{~s}$ per scan. At the impulsive phase, the HXT saw emission up to its highest-energy channel $(53-93 \mathrm{keV})$ : see the light curves at four energy bands in Figure 4 (left-hand panel). The HXT images show the double-footpoint emission characteristic of large flares with soft X-ray emission (from the Yohkoh Soft X-ray Telescope) in between (see right-hand panel of Figure 4).

In Figure 5, channel 4 spectra over the Ca XIX lines are indicated during the 25 August 2001 flare with forward and backward scans (wavelength increasing and decreasing with time, respectively).

\subsection{Plasma Dynamics in the 25 August 2001 Flare}

Velocities of the emitting plasma during the 25 August 2001 flare were found from DIOGENESS Si XIII, S XV, and Ca XIX spectra, with the Ca XIX spectra from the dopplerometer part of the instrument. The left panel of Figure 6 shows the Ca XIX spectra in channels 1 and 4 plotted in stepper motor addresses. The main lines apparent are the resonance $(w)$, intercombination $(x, y)$, and forbidden $(z)$ lines of Ca XIX in order of wavelength (see Table 3). The channel 1 spectrum (in blue in Figure 6) has wavelength increasing to the left, the channel 4 spectrum (in red) with channel 1 to the right. The scan times of the spectra are almost coincident. The deduced velocities for all channels are given as a function of time in Figure 6 (right panel). They indicate that there were high approach velocities at 

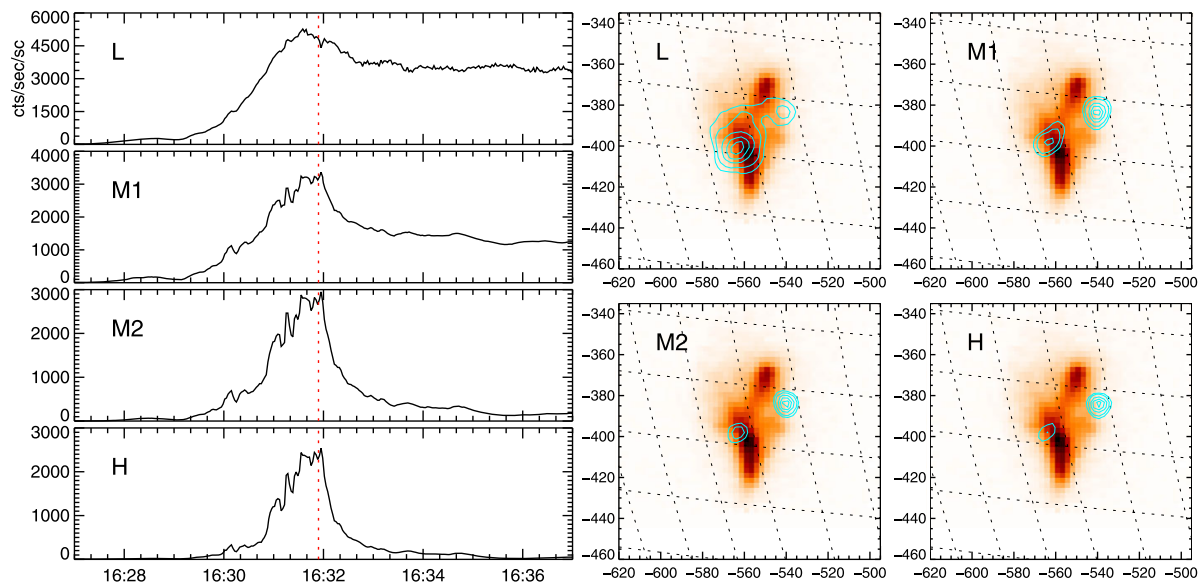

Figure 4 Left: Light curves over the impulsive phase of the 25 August 2001 flare from the Yohkoh HXT instrument in its four channels: L $-14-23 \mathrm{keV}, \mathrm{M} 1-23-33 \mathrm{keV}, \mathrm{M} 2-33-53 \mathrm{keV}, \mathrm{H}-53-93 \mathrm{keV}$. Right: Yohkoh SXT images (red intensity scale) and HXT images (blue contours) in the four channels of HXT indicated at the top of each image.
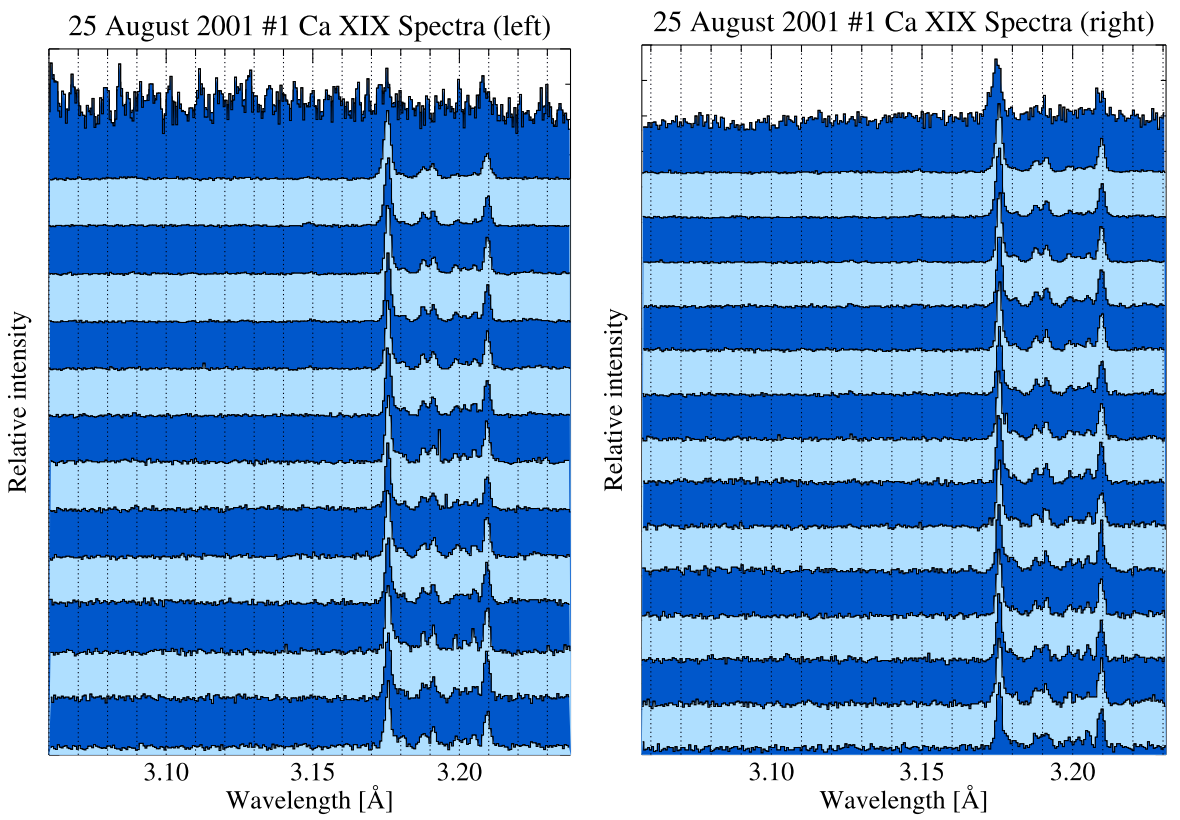

Figure 5 Left: Forward (increasing wavelength) scans for DIOGENESS channel 4 (Ca XIX lines) during the 25 August 2001 flare stacked with increasing times from top to bottom (time range 16:29 - 18:00 UT). Right: Corresponding backward (decreasing wavelength) scans.

the time (approximately $27-16: 29$ UT) at the start of the hard X-ray impulses recorded by the Yohkoh HXT M1, M2, and H channels (see light curves and images in Figure 4). The highest velocity, $165 \mathrm{~km} \mathrm{~s}^{-1}$, is recorded by the $\mathrm{S} \mathrm{XV}$ (temperature $\sim 10 \mathrm{MK}$ ) line emission. The velocity of the higher temperature $(\sim 20 \mathrm{MK})$ Ca XIX line emission indicates a velocity 

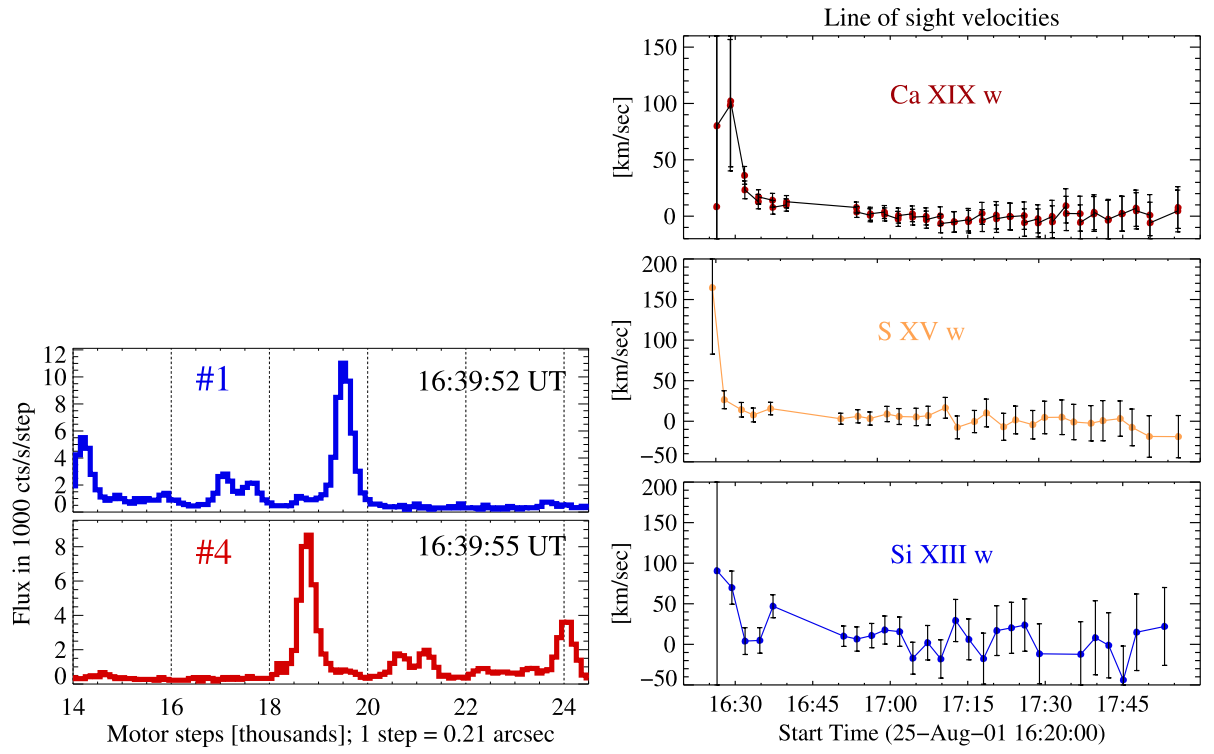

Figure 6 Left: Ca XIX spectra from channels 1 and 4 (reversed), plotted as a function of stepper motor addresses, for two times (indicated) in the 25 August 2001 flare. Right: Line-of-sight velocities of X-ray flare plasma as deduced from Doppler shifts of Ca XIX, S XV, and Si XIII lines during the flare of 25 August 2001. See Płocieniak et al. (2002) for details.

of about $100 \mathrm{~km} \mathrm{~s}^{-1}$, as does $\left(\approx 90 \mathrm{~km} \mathrm{~s}^{-1}\right)$ the lower temperature $(\sim 7 \mathrm{MK})$ Si XIII line emission.

These velocities are smaller than those previously observed for large disk flares. Doschek et al. (1980), who discussed several X-class flares seen with X-ray spectrometers on the P78-1 spacecraft, found approach velocities of about $400 \mathrm{~km} \mathrm{~s}^{-1}$ for disk flares, as did Tanaka et al. (1982) for a very large disk flare seen with an X-ray spectrometer on the Hinotori spacecraft. It is possible that plasma motions during the 25 August 2001 flare observed by DIOGENESS were directed at a substantial angle to the line of sight (although the flare was on the disk, it was $34^{\circ}$ east of the central meridian), which would account for the lower observed approach velocities in its rise phase.

\subsection{Spectral Line Identifications}

Figure 7 shows an averaged spectrum from DIOGENESS channels 1 and 4 during the $25 \mathrm{Au}-$ gust 2001 flare, peak time in soft X-rays at 16:45 UT (Table 2), with flux on a logarithmic scale to show the weak line emission either side of the Ca XIX line emission to better advantage. Table 3 gives the wavelengths of identifiable line features for the $3.05-3.30 \AA$ range. Channels 1 and 4 spectra cover the region of the Ca XIX resonance $(w)$ line and include other Ca XIX lines and dielectronic satellites of the Li-like (Ca XVIII) and lower stages of ionization. These lines are familiar from previously flown X-ray spectrometers on the $P 78-1$, SMM, Hinotori, and Yohkoh spacecraft. The ratios of the principal satellites ( $k$ and the $d 13$, $d 15$ lines) to the Ca XIX $w$ line have been used extensively to find flare and non-flaring active region temperatures and emission measures, based on the theory of Gabriel (1972) (whose line notation we use here) and Bely-Dubau et al. (1982). Ca XVIII satellite $j$ is indistinguishable from the Ca XIX line $z$. The ratio of the inner-shell excitation satellite $q$ to 
Figure 7 Averaged DIOGENESS spectrum from channels 1 and 4 during the 25 August 2001 flare showing Ar XVII and Ca XIX lines and dielectronic satellites of Ca XVIII, Ca XVII, and Ca XVI.

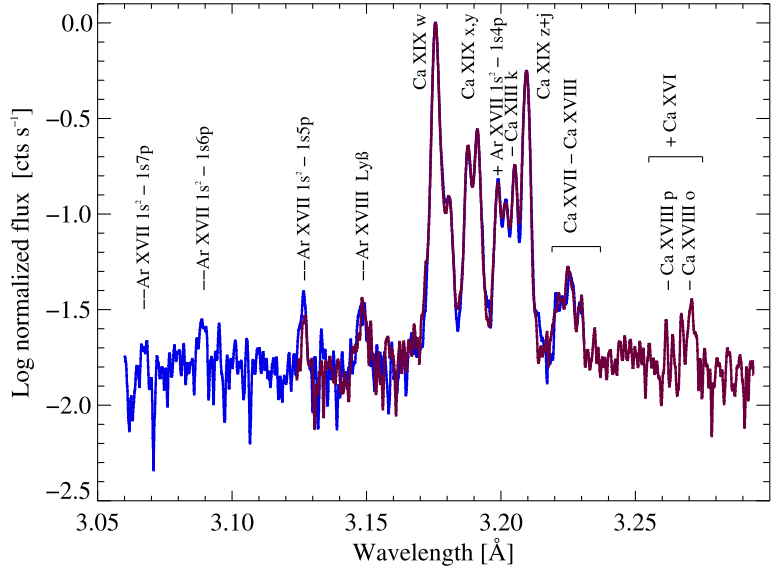

line $w$ depends on the ion fraction $N\left(\mathrm{Ca}^{+17}\right) / N\left(\mathrm{Ca}^{+18}\right)$ and therefore in principle is a means of finding departures of ionization equilibrium in the emitting plasma. However, satellite $q$ is blended with the Ar XVII line $w 4$ (this notation being used for the transition $1 s^{2}-1 s 4 p$ ).

In the DIOGENESS spectrum shown in Figure 7, the Rydberg series of Ar XVII lines up to the $w 7$ line can be discerned. The Ca XIX and Ar XVII spectra up to the $w 10$ line have been observed during strong flares with crystal spectrometers on the P78-1 spacecraft (Doschek, Feldman, and Seely, 1985; Seely and Feldman, 1985), with lines $w 9$ and $w 10$ blended with Ca XIX dielectronic satellites on the long-wavelength side of the Ca XX Ly$\alpha$ doublet. Spectra from the Alcator C-Mod tokamak plasmas, recently reported by Rice et al. (2014), also show Ar XVII high- $n$ lines up to the ionization limit at $3.009 \AA$. All these lines, which are shortward of $3.06 \AA$, are outside the DIOGENESS range. We note that the estimated temperatures $(\sim 30 \mathrm{MK})$ and electron densities $\left(\sim 10^{15} \mathrm{~cm}^{-3}\right)$ in the Alcator plasmas are both higher than those typical of large solar flare plasmas $(\sim 25 \mathrm{MK}$ and $\sim 10^{11}-10^{12} \mathrm{~cm}^{-3}$, respectively), but this has little effect on the appearance of the spectral lines, whose the principal excitation mechanism is electron collisional excitation from the ground state of ions in both cases.

In Table 3, we follow Rice et al. (2014) and Seely and Doschek (1989) in identifying lines in the DIOGENESS spectrum on the long-wavelength side of the Ca XIX forbidden line $z$ as Ca XVII satellites including the strong inner-shell-excited line $\beta$, as well as Ca XVIII satellites $u$ and $v$, which are both dielectronically and collisionally excited (Gabriel, 1972). In addition, a feature possibly composed of several lines between $3.261 \AA$ and $3.272 \AA$, not apparently identified before, is probably caused by a group of Ca XVI lines by analogy with equivalent lines of Fe XXII seen in solar flare spectra with the SMM Bent Crystal Spectrometer (Phillips et al., 1983). The Ca XVIII two-electron-jump satellites $o$ and $p$ (transitions $1 s^{2} 2 p^{2} P_{3 / 2}-1 s 2 s^{2} S_{1 / 2}, 1 s^{2} 2 p^{2} P_{1 / 2}-1 s 2 s^{2} S_{1 / 2}$ ) may also contribute to this line feature: their wavelengths are estimated by Bely-Dubau et al. (1982) to be $3.2688 \AA$ and $3.2636 \AA$, respectively.

In DIOGENESS channel 2 spectra, the predominant lines are the resonance, intercombination, and forbidden lines of S XV and the Ly- $\beta(1 s-3 p)$ line of Si XIV, prominent also in RESIK spectra. The $x$ and $y$ lines are partially blended as are S XIV satellites $d 13, d 15$ with $\mathrm{S} X V$ line $w$ and $\mathrm{S} X I V$ satellites $j, k$ with $\mathrm{S}$ XV line $z$. There is a possible long-wavelength "shoulder" of emission to the Si XIV line, which may be due to the Si XIII $w 6$ line. In channel 3 spectra, the Si XIII $w-z$ lines are the most prominent with Si XII satellites $d 13+d 15$, 
Table 3 Spectral line identifications in DIOGENESS spectra.

\begin{tabular}{|c|c|c|c|c|c|}
\hline \multirow{2}{*}{$\begin{array}{l}\text { Measured line } \\
\lambda(\AA)\end{array}$} & \multicolumn{5}{|c|}{ Line identification } \\
\hline & Ion & $\lambda(\AA)$ & Transition & Name $^{\mathrm{a}}$ & Source $^{\mathrm{b}}$ \\
\hline \multicolumn{6}{|c|}{ Channels 1 and 4} \\
\hline \multicolumn{5}{|c|}{ 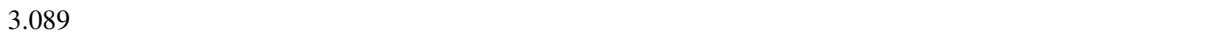 } & $\mathrm{R}, \mathrm{S}$ \\
\hline 3.095 & Ar XVII & 3.095 & $1 s^{2}{ }^{1} S_{0}-1 s 6 p^{1} P_{1}$ & $w 6$ & $\mathrm{~K}, \mathrm{~S}$ \\
\hline 3.127 & Ar XVII & 3.128 & $1 s^{2}{ }^{1} S_{0}-1 s 5 p^{1} P_{1}$ & $w 5$ & $\mathrm{~K}, \mathrm{~S}$ \\
\hline \multicolumn{6}{|l|}{3.148} \\
\hline 3.177 & Ca XIX & 3.177 & $1 s^{2}{ }^{1} S_{0}-1 s 2 p{ }^{1} P_{1}$ & $w$ & $\mathrm{~K}$ \\
\hline 3.181 & Ca XVIII & 3.1809 & $1 s^{2} 3 p^{2} P_{3 / 2}-1 s 2 p\left({ }^{1} P\right) 3 p^{2} D_{5 / 2}$ & $d 13$ & K \\
\hline 3.188 & Ca XIX & 3.1889 & $1 s^{21} S_{0}-1 s 2 p^{3} P_{2}$ & $x$ & $\mathrm{~K}$ \\
\hline 3.191 & Ca XIX & 3.1925 & $1 s^{2}{ }^{1} S_{0}-1 s 2 p^{3} P_{1}$ & $y$ & K \\
\hline \multirow[t]{2}{*}{3.199} & Ca XVIII & 3.2003 & $1 s^{2} 2 s^{2} S_{1 / 2}-1 s\left(2 s 2 p^{3} P\right)^{2} P_{3 / 2}$ & $q$ & K \\
\hline & Ar XVII & 3.200 & $1 s^{2}{ }^{1} S_{0}-1 s 4 p^{1} P_{1}$ & $w 4$ & K \\
\hline 3.203 & Ca XVIII & 3.2031 & $1 s^{2} 2 s^{2} S_{1 / 2}-1 s\left(2 s 2 p^{3} P\right)^{2} P_{1 / 2}$ & $r$ & K \\
\hline 3.206 & Ca XVIII & 3.2058 & $1 s^{2} 2 p^{2} P_{1 / 2}-1 s 2 p^{22} D_{3 / 2}$ & $k$ & K \\
\hline \multirow[t]{2}{*}{3.210} & Ca XIX & 3.2111 & $1 s^{21} S_{0}-1 s 2 s^{3} S_{1}$ & $z$ & K \\
\hline & Ca XVIII & 3.2097 & $1 s^{2} 2 p^{2} P_{3 / 2}-1 s 2 p^{22} D_{5 / 2}$ & $j$ & K \\
\hline 3.222 & Ca XVII & 3.2217 & $1 s^{2} 2 s^{21} S_{0}-1 s 2 s^{2} 2 p^{1} P_{1}$ & $\beta$ & $\mathrm{R}$ \\
\hline 3.225 & Ca XVII & $?$ & $1 s^{2} 2 s^{2}-1 s 2 s^{2} 2 p$ & & $\mathrm{Ph}$ \\
\hline 3.225 & Ca XVIII & 3.2266 & $1 s^{2} 2 s^{2} S_{1 / 2}-1 s 2 s 2 p^{4} P_{3 / 2}$ & $u$ & $\mathrm{R}, \mathrm{D}$ \\
\hline 3.230 & Ca XVIII & 3.2277 & $1 s^{2} 2 s^{2} S_{1 / 2}-1 s 2 s 2 p^{4} P_{1 / 2}$ & $v$ & $\mathrm{R}, \mathrm{D}$ \\
\hline 3.230 & Ca XVII & $?$ & $1 s^{2} 2 s^{2}-1 s 2 s^{2} 2 p$ & & $\mathrm{Ph}$ \\
\hline 3.272 & Ca XVI & $?$ & $1 s^{2} 2 s^{2} 2 p-1 s 2 s 2 p^{2}$ & & $\mathrm{Ph}$ \\
\hline \multicolumn{6}{|l|}{ Channel 2} \\
\hline 5.037 & $\mathrm{~S} \times \mathrm{V}$ & 5.0385 & $1 s^{2}{ }^{1} S_{0}-1 s 2 p{ }^{1} P_{1}$ & $w$ & $\mathrm{~K}$ \\
\hline 5.047 & S XIV & 5.0468 & $1 s^{2} 3 p^{2} P_{3 / 2}-1 s 2 p\left({ }^{1} P\right) 3 p^{2} D_{5 / 2}$ & $d 13$ & K \\
\hline 5.062 & $\mathrm{~S} X \mathrm{~V}$ & $5.06 ?$ & $1 s^{21} S_{0}-1 s 2 p^{3} P_{2}$ & $x$ & K \\
\hline 5.064 & $\mathrm{Sxv}$ & 5.0662 & $1 s^{21} S_{0}-1 s 2 p^{3} P_{1}$ & $y$ & $\mathrm{~K}$ \\
\hline 5.083 & S XIV & 5.0861 & $1 s^{2} 2 s^{2} S_{1 / 2}-1 s\left(2 s 2 p^{3} P\right)^{2} P_{3 / 2}$ & $q$ & K \\
\hline \multirow[t]{4}{*}{5.099} & S XIV & 5.0964 & $1 s^{2} 2 p^{2} P_{1 / 2}-1 s 2 p^{2} D_{3 / 2}$ & $k$ & K \\
\hline & $\mathrm{S} \times \mathrm{V}$ & 5.101 & $1 s^{2}{ }^{1} S_{0}-1 s 2 s^{3} S_{1}$ & $z$ & K \\
\hline & S XIV & 5.1010 & $1 s^{2} 2 p^{2} P_{3 / 2}-1 s 2 p^{22} D_{5 / 2}$ & $j$ & $\mathrm{~K}$ \\
\hline & Si XIV & 5.217 & $1 s^{2} S_{1 / 2}-2 p^{2} P_{1 / 2,3 / 2}$ & Ly- $\beta$ & \\
\hline \multicolumn{6}{|l|}{ Channel 3} \\
\hline 6.410 & Mg XII ? & 6.497 & $1 s^{2} S_{1 / 2}-8 p^{2} P_{1 / 2,3 / 2}$ & Ly- $\eta$ & $\mathrm{K}$ \\
\hline 6.490 & $\mathrm{Mg}$ XII ? & 6.497 & $1 s^{2} S_{1 / 2}-6 p^{2} P_{1 / 2,3 / 2}$ & Ly- $\epsilon$ & $\mathrm{K}$ \\
\hline \multicolumn{6}{|l|}{6.513} \\
\hline \multicolumn{6}{|l|}{6.545} \\
\hline 6.574 & Mg XII ? & 6.580 & $1 s^{2} S_{1 / 2}-5 p^{2} P_{1 / 2,3 / 2}$ & Ly- $\delta$ & $\mathrm{K}$ \\
\hline 6.641 & Si XIII & 6.6477 & $1 s^{21} S_{0}-1 s 2 p^{1} P_{1}$ & $w$ & $\mathrm{~K}$ \\
\hline \multicolumn{6}{|l|}{6.681} \\
\hline 6.686 & Si XIII & 6.6879 & $1 s^{21} S_{0}-1 s 2 p^{3} P_{1}$ & $y$ & $\mathrm{~K}$ \\
\hline 6.713 & Si XII & 6.718 & $1 s^{2} 2 s^{2} S_{1 / 2}-1 s\left(2 s 2 p^{3} P\right)^{2} P_{3 / 2}$ & $q$ & K \\
\hline 6.737 & Si XIII & 6.740 & $1 s^{2} S_{0}-1 s 2 s^{3} S_{1}$ & $z$ & $\mathrm{~K}$ \\
\hline
\end{tabular}

${ }^{a}$ Gabriel (1972) for He-like ion $1 s^{2}-1 s 2 l$ transitions and Li-like ion satellites.

${ }^{b} \mathrm{D}=$ Doschek et al. (1985); K = Kelly (1987); R = Rice et al. (2014); $\mathrm{Ph}=$ Phillips et al. (1983); $\mathrm{S}=$ Seely and Feldman (1985). 
Table 4 ChemiX Spectral Channels.

\begin{tabular}{|c|c|c|c|c|c|}
\hline $\begin{array}{l}\text { Channel } \\
\text { No. }\end{array}$ & Crystal & $\begin{array}{l}\text { Diffracting } \\
\text { plane }\end{array}$ & $\begin{array}{l}2 d \\
(\AA)\end{array}$ & $\begin{array}{l}\text { Wavelength } \\
\text { Range (§) }\end{array}$ & $\begin{array}{l}\text { Av. spectral } \\
\text { resolution } \\
(\mathrm{m} \AA \text { per pixel) }\end{array}$ \\
\hline \multicolumn{6}{|c|}{ Spectrometer channels } \\
\hline 1 & $\mathrm{Si}$ & 111 & 6.271 & $1.500-2.713$ & 1.46 \\
\hline 2 & Quartz & $10 \overline{1} 0$ & 8.514 & $2.700-4.304$ & 1.95 \\
\hline 3 & KDP & 011 & 10.185 & $4.290-5.228$ & 1.43 \\
\hline 4 & KAP & 001 & 26.64 & $5.200-8.800$ & 4.73 \\
\hline \multicolumn{6}{|c|}{ Dopplerometer channels } \\
\hline 1 & $\mathrm{LiF}$ & 022 & 2.848 & $1.830-1.925$ & 0.10 \\
\hline 2 & $\mathrm{Si}$ & 111 & 6.271 & $3.150-3.245$ & 0.10 \\
\hline 3 & $\mathrm{Si}$ & 111 & 6.271 & $3.90-4.080$ & 0.19 \\
\hline
\end{tabular}

$q$, and $k$ identifiable. Possible very weak line features to the short-wavelength side of the Si XIII line $w$ occur. The Mg XII Rydberg sequence $1 s-n p$ occur in this region and were seen with the crystal spectrometers on OSO-8 (Parkinson et al., 1978). They may account for the $6.410 \AA(n=8), 6.490 \AA(n=6)$, and $6.580 \AA(n=5)$ lines in the DIOGENESS spectra, although the $n=7 \mathrm{Mg}$ XII line at $6.448 \AA$ is not apparent. The $n=4 \mathrm{Mg}$ XII line blends with the Si XIII $z$ line. Note that the anomaly in the effective area due to the beryl crystal reflectivity at about $6.75 \AA$ (see Figure 2) distorts the intensity of the Si XIII $z$ line.

In time, the line identifications given in Table 3 will be refined with better atomic data. In particular, we plan to run atomic codes to find the wavelengths of the satellites due to Ca XVI, as was done for the case of the equivalent Fe ions (Phillips et al., 1983).

\section{The ChemiX Instrument for Interhelioprobe}

ChemiX (CHEMical composition In X-rays) is a bent crystal spectrometer under construction that will observe the soft X-ray spectra of solar flares, continuing the objectives of previous spectrometers such as the Flat Crystal Spectrometer and Bent Crystal Spectrometer on Solar Maximum Mission (Acton et al., 1980), SOLEX and SOLFLEX on P78-1 (Doschek, Kreplin, and Feldman, 1979), and RESIK and DIOGENESS on CORONAS-F. We briefly describe it here, while a more detailed account is in preparation (Siarkowski et al.). ChemiX will have four "spectrometer" channels viewing spectral lines of Fe XXV, Ca XIX, S XV, Si XIII and Si XIV, and three pairs of crystals in a dopplerometer arrangement like that on DIOGENESS and its predecessors, viewing lines of Fe XXV, Ca XIX, and Ar XVII. There will be important improvements over both the RESIK and DIOGENESS instruments in that the detectors will be X-ray-sensitive, cooled (to $<-20^{\circ} \mathrm{C}$ ) charge-coupled devices (CCDs), and there will be a two-dimensional collimator with $\sim 3 \operatorname{arcmin}(\mathrm{FWHM})$ field of view that will enable the instruments to view single active regions and so avoid possible spatial and spectral confusion for the spectrometer channels. A projected 1-s time resolution will allow the highly dynamic impulsive phases of flares to be observed, in particular changes to spectral line profiles and shifts indicative of plasma turbulence and motions. Table 4 gives details of the Bragg-diffracting crystals to be used and the wavelength ranges in each of the seven channels. There will be full spectral coverage in the range $1.5-9 \AA$ with a spectral resolution 


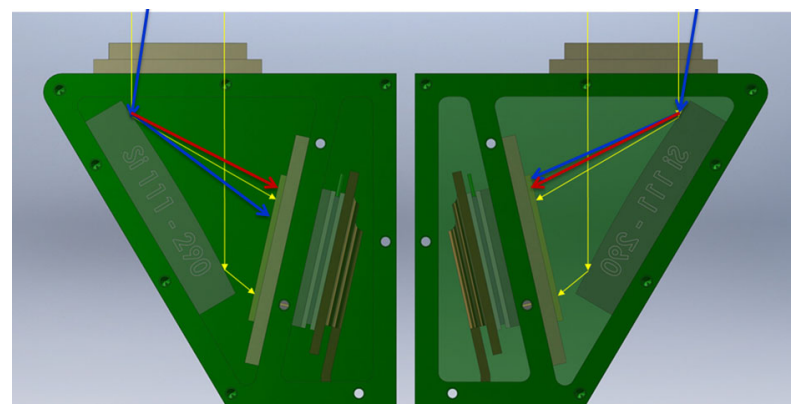

Figure 8 Scheme of ChemiX. Solar X-rays are incident from the top of the figure (blue, yellow, and red arrows), are diffracted off the two bent crystals ( $\mathrm{Si} 111$ in both cases), and are then incident on the CCDs. The yellow rays are for a source in the centre of the collimator field of view (FOV), the blue rays from a source moved across the FOV (tangential motion), and the red rays for a source approaching the instrument (i.e., subject to a Doppler shift).

that is expected to be four to ten times better than that of RESIK because of the use of CCDs over gas proportional counters. The signal-to-noise ratio will also be much improved, and because of the use of crystals with low atomic number, there will be very little instrumental background due to crystal fluorescence, enabling the solar continuum to be measured. Figures 8 and 9 give the scheme for ChemiX, which is at present under construction.

Other subsystems of ChemiX include an energetic particle (electrons, protons, and alphaparticles) detector, so that the instrument will have its own monitor for safety concerns with the electronics; a soft X-ray pin-hole camera with CCD detector, providing X-ray context images with one-arcmin spatial resolution that will help to ascertain which active region a flare originated from; and a movable target-pointing platform that can lock the spectrometer and CCDs on to a particular flare or other region within seconds of command from a flare trigger. Spectral atlases consisting of full spectral scans over the entire 1.5-9 Å range will be taken while the instrument is directed at particular sources with a user-chosen intensity level (e.g. a total of 10000 photon counts over the spectrum). Spectral line and continuum fluxes will be obtained with unprecedented precision, especially when the spacecraft is at perihelion, about $0.3 \mathrm{AU}$ from the Sun. Thermal filters over the instrument entrance aperture will prevent solar ultraviolet radiation from entering.

\section{Conclusions}

The DIOGENESS instrument on CORONAS-F operated for only a few weeks in 2001, but several flares with GOES importance of up to X5 were recorded. Its scanning crystal spectrometers observed the neighbourhood of the He-like $\mathrm{Ca}, \mathrm{S}$, and $\mathrm{Si}$ (Ca XIX, S XV, and Si XIII) X-ray emission lines at $3.17 \AA, 5.04 \AA$, and $6.65 \AA$, with the two quartz crystals of channels 1 and 4 in a dopplerometer mode, i.e. arranged in such a way that the Doppler line shifts at flare impulsive phases could be separated from spatial shifts. The concept was validated for the X5 flare on 25 August 2001, in which velocities of up to $150 \mathrm{~km} \mathrm{~s}^{-1}$ were observed. The strong X-ray emission during this flare enabled many lines to be distinguished and identified. The Ca XIX lines predominate in channels 1 and 4 spectra, with dielectronic satellites of Ca XVIII, Ca XVII, and Ca XVI also present. The spectra are similar to those recorded with the X-ray spectrometer on the P78-1 spacecraft (Doschek, Feldman, and Seely, 1985; Seely and Feldman, 1985), and resemble to a high degree those obtained from 

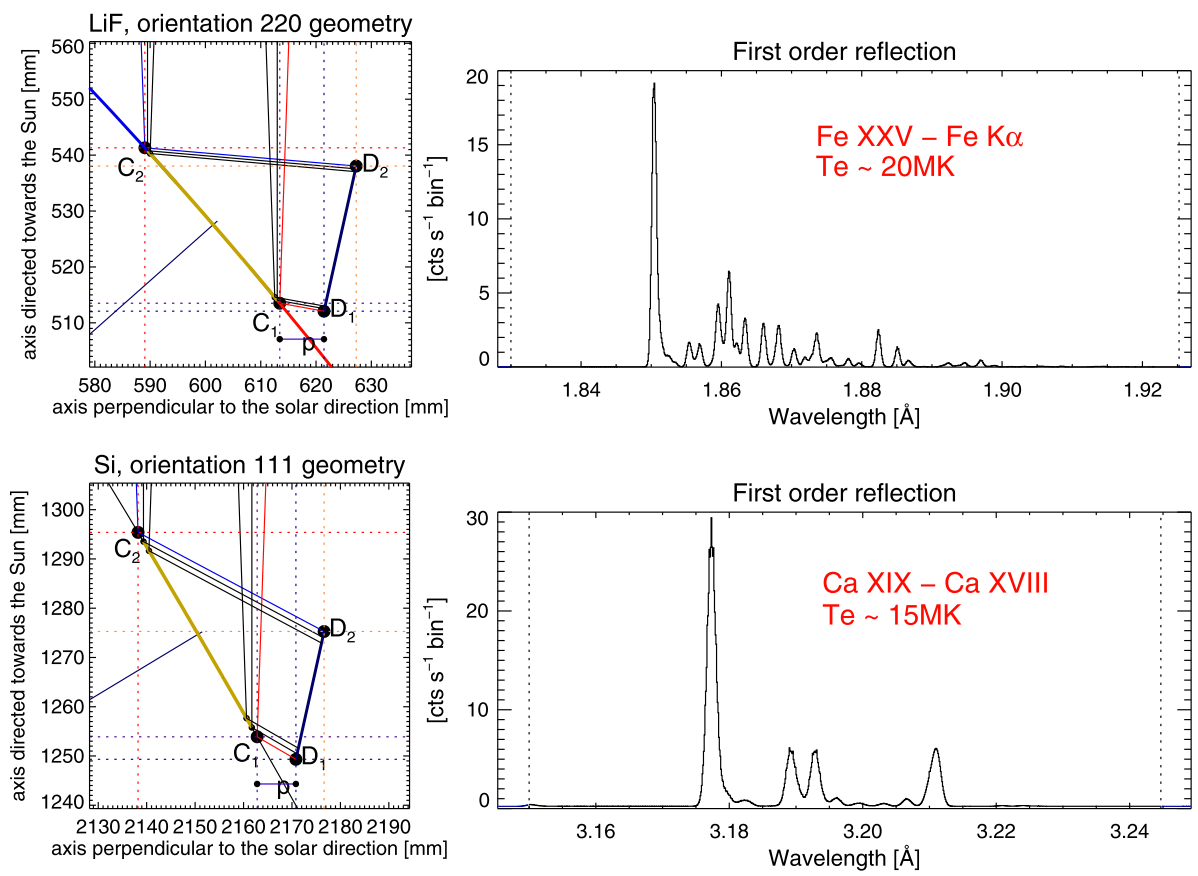

Figure 9 Scheme of crystal diffraction for dopplerometer channels 1 (Fe XXV, top) and 2 (Ca XIX, lower) and resulting spectra in ChemiX (simulated for a GOES M5 flare using the CHIANTI database and software package). Left: Solar X-rays are incident from the top of the figure on the crystal (light green), are diffracted by the crystals $\left(C_{1}-C_{2}\right)$, and then are incident on the CCD detectors $\left(D_{1}-D_{2}\right)$. Right: Simulated spectra calculated for a Sun-spacecraft distance of 1 AU using the CHIANTI database - top panel is the Fe XXV spectrum with Fe XXIV dielectronic satellites, lower panel is the Ca XIX spectrum with relatively much weaker Ca XVIII satellites. Temperatures (in MK) are indicated in the figure legend.

the Alcator C-Mod tokamak (Rice et al., 2014), including the Rydberg series of Ar XVII $1 s^{2}-1 s n p$ lines, which are seen up to $n=7$ in DIOGENESS spectra. In channel 2 and 3 spectra, lines of S XV, Si XIII, and Si XIV predominate, with dielectronic satellites of the Li-like stages occurring.

The ChemiX instrument on the forthcoming Russian Interhelioprobe spacecraft, which will be launched into elliptical orbits around the Sun in 2020 and 2022, will have four channels viewing the Fe XXV, Ca XIX, S XV, Si XIII, and Si XIV X-ray emission lines, with a full coverage of the $1.5-9 \AA$ range. The diffracting crystals will be arranged in a dopplerometer manner as for DIOGENESS, but will be bent and non-moving, not flat and scanning. This will be a considerable improvement in that the time resolution should be much higher. The use of cooled CCD detectors will also give a much better spectral resolution than did the RESIK spectrometer on CORONAS- $F$, which had position-sensitive proportional counters. A collimator will allow the emission from individual active regions to be observed.

Acknowledgements We acknowledge support from the Polish National Science Centre grants 2011/01/B/ ST9/05861, 4 T12E 045 29, 2011/01/M/ST9/06096, 2314/7.PR/2012/2 and 2011/01/M/ST9/05878. The research leading to these results has received funding from the European Union's Seventh Programme for Research, Technological Development and Demonstration under Grant Agreement No. 284461 (eHEROES project). We also thank the Rutherford Appleton Laboratory (UK) for providing the beryl crystal of DIOGENESS, which was a spare crystal from constructing the Solar Maximum Mission Flat Crystal Spectrome- 
ter. The CHIANTI atomic database and code is a collaborative project involving the George Mason University, the University of Michigan (USA), and the University of Cambridge (UK).

Open Access This article is distributed under the terms of the Creative Commons Attribution License which permits any use, distribution, and reproduction in any medium, provided the original author(s) and the source are credited.

\section{References}

Acton, L.W., Finch, M.L., Gilbreth, C.W., Culhane, J.L., Bentley, R.D., Bowles, J.A., Guttridge, P., Gabriel, A.H., Firth, J.G., Hayes, R.W.: 1980, The soft X-ray polychromator for the Solar Maximum Mission. Solar Phys. 65, 53. DOI.

Bely-Dubau, F., Faucher, P., Steenman-Clark, L., Dubau, J., Loulergue, M., Gabriel, A.H., Antonucci, E., Volonte, S., Rapley, C.G.: 1982, Dielectronic satellite spectra for highly-charged helium-like ions. VII Calcium spectra: Theory and comparison with SMM observations. Mon. Not. Roy. Astron. Soc. 201, 1155 .

Culhane, J.L., Hiei, E., Doschek, G.A., Cruise, A.M., Ogawara, Y., Uchida, Y., Bentley, R.D., Brown, C.M., Lang, J., Watanabe, T., Bowles, J.A., Deslattes, R.D., Feldman, U., Fludra, A., Guttridge, P., Henins, A., Lapington, J., Magraw, J., Mariska, J.T., Payne, J., Phillips, K.J.H., Sheather, P., Slater, K., Tanaka, K., Towndrow, E., Trow, M.W., Yamaguchi, A.: 1991, The Bragg crystal spectrometer for SOLAR-A. Solar Phys. 136, 89. DOI.

Doschek, G.A., Feldman, U., Kreplin, R.W., Cohen, L.: 1980, High-resolution X-ray spectra of solar flares. III - General spectral properties of X1-X5 type flares. Astrophys. J. 239, 725. DOI.

Doschek, G.A., Feldman, U., Seely, J.F.: 1985, Element abundances from solar flare spectra. Mon. Not. Roy. Astron. Soc. 217, 317.

Doschek, G.A., Kreplin, R.W., Feldman, U.: 1979, High-resolution solar flare X-ray spectra. Astrophys. J. Lett. 233, L157. DOI.

Gabriel, A.H.: 1972, Dielectronic satellite spectra for highly-charged helium-like ionlines. Mon. Not. Roy. Astron. Soc. 160, 99.

Oraevsky, V.N., Sobelman, I.I.: 2002, Comprehensive studies of solar activity on the CORONAS-F satellite. Astron. Lett. 28, 401. DOI.

Parkinson, J.H., Wolff, R.S., Kestenbaum, H.L., Ku, W.H.M., Lemen, J.R., Long, K.S., Novick, R., Suozzo, R.J., Weisskopf, M.C.: 1978, Silicon X-ray line emission from solar flares and active regions. Solar Phys. 60, 123. DOI.

Phillips, K.J.H., Lemen, J.R., Cowan, R.D., Doschek, G.A., Leibacher, J.W.: 1983, Inner-shell transitions in Fe XIX - XXII in the X-ray spectra of solar flares and Tokamaks. Astrophys. J. 265, 1120. DOI.

Płocieniak, S., Sylwester, J., Kordylewski, Z., Sylwester, B.: 2002, Determination of wavelengths and line shifts based on X-ray spectra from Diogeness. In: Wilson, A. (ed.) Solar Variability: From Core to Outer Frontiers, ESA SP-506, 963.

Rice, J.E., Reinke, M.L., Ashbourn, J.M.A., Gao, C., Victora, M.M., Chilenski, M.A., Delgado-Aparicio, L., Howard, N.T., Hubbard, A.E., Hughes, J.W., Irby, J.H.: 2014, X-ray observations of $\mathrm{Ca}^{19+}, \mathrm{Ca}^{18+}$ and satellites from Alcator C-Mod tokamak plasmas. J. Phys. B, At. Mol. Opt. Phys. 47(7), 075701. DOI.

Seely, J.F., Doschek, G.A.: 1989, Measurement of wavelengths for inner-shell transitions in CA XVII - XIX. Astrophys. J. 338, 567. DOI.

Seely, J.F., Feldman, U.: 1985, Precision measurement of transition energies and Lamb shifts in two-electron argon and potassium in solar-flare spectra. Phys. Rev. Lett. 54, 1016. DOI.

Siarkowski, M., Sylwester, J., Plocieniak, S., Kordylewski, Z.: 2002, Flare X-ray spectra observed by Diogenes scanning Bragg spectrometer. In: Wilson, A. (ed.) Solar Variability: From Core to Outer Frontiers, ESA SP-506, 753.

Sylwester, B., Sylwester, J., Phillips, K.J.H., Kȩpa, A., Mrozek, T.: 2014, Solar flare composition and thermodynamics from RESIK X-ray spectra. Astrophys. J. 787, 122. DOI.

Sylwester, J., Gaicki, I., Kordylewski, Z., Kowaliński, M., Nowak, S., Płocieniak, S., Siarkowski, M., Sylwester, B., Trzebiński, W., Bakała, J., Culhane, J.L., Whyndham, M., Bentley, R.D., Guttridge, P.R., Phillips, K.J.H., Lang, J., Brown, C.M., Doschek, G.A., Kuznetsov, V.D., Oraevsky, V.N., Stepanov, A.I., Lisin, D.V.: 2005, RESIK: a bent crystal X-ray spectrometer for studies of solar coronal plasma composition. Solar Phys. 226, 45. DOI.

Tanaka, K., Watanabe, T., Nishi, K., Akita, K.: 1982, High-resolution solar flare X-ray spectra obtained with rotating spectrometers on the HINOTORI satellite. Astrophys. J. Lett. 254, L59. DOI. 